Ann. Sci. forest., 1981, 38 (2), 223-236.

\title{
Application des données de l'inventaire forestier national à l'étude de la production du Pin sylvestre en Margeride
}

\section{A. - Etude de la croissance en hauteur}

\author{
J.-M. OTTORINI* et C. NYS** \\ * Station de Sylviculture ef de Production \\ **: Station de Recherches sur les Sols forestiers et la Fertilisation \\ Centre de Recherches forestieres de Nancy, I.N.R.A., \\ Champenoux, 54280 Seichamps
}

\begin{abstract}
Résumé
A l'occasion d'une étude socio-économique de la Margeride dans le Massif Central, le besoin s'est présenté de connaître la production du Pin sylvestre (Pinus sylvestris) dans cette région. Pour éviter la charge de nouvelles campagnes de mesures, des donnćes provenant de l'Inventaire Forestier National furent utilisées. Cet article présente la première partie de l'étude dans laquelle les courbes caractéristiques de la croissance en hauteur pour les stations de différentes productivités furent estimées. Comme principal résultat, la hauteur moyenne des arbres dominants et codominants des placettes put être directement estimée par une fonction de l'âge et de l'altitude en utilisant la régression non linéaire. Des difficultés étant apparues avec une dispersion non homogène de la variable dépendante, des solutions pratiques faisant un certain appel à l'intuition furent proposées.
\end{abstract}

\section{I. - Introduction}

La participation de laboratoires du C.N.R.F. à une Action Concertée de la D.G.R.S.T. (1) pour rechercher les moyens de mettre en valeur une région agricole du Massif Central (la Margeride) a été l'occasion de poser un problème nouveau et intéressant : il s'agissait d’étudier la production d'une essence forestière importante de la région (le Pin syivestre) à laide de données disponibles de l'Inventaire Forestier National, de façon à éviter de peser sur le potentiel technique des laboratoires en question.

Limportance de la hauteur d'un peuplement équienne et de son évolution au cours du temps dans les études de production est bien établie, comme peuvent

(1) Responsable scientifique : L. Gachon, I.N.R.A., Département d'agronomic, 12, avenue de l'Agriculture, 63100 Clermont-Ferrand. 
l'attester les articles qui seront cités et la vaste littérature sur laquelle ils s'appuient. La relation entre la hauteur et l'âge peut procurer une excellente évaluation de la production totale à un moment donné par lintermédiaire d’autres relations adéquates. La hauteur d'un peuplement (hauteur moyenne des arbres qui le composent ou hauteur moyenne de certains des plus hauts) caractérise si bien sa productivité que la hauteur à un âge de référence (souvent 50 ans) est souvent assimilée à cette productivité, alors que celle-ci devrait être exprimée en volume total à un âge de référence. Pour ces raisons nos efforts se sont d'abord adressés à l'étude de la croissance en hauteur. Compte tenu de la taille des placettes de I'Inventaire Forestier National qui sont assez petites (en moyenne 2,5 ares), un compromis a été réalisé entre les deux définitions habituellement utilisées : la hauteur dominante (qui caractérise le mieux la productivité du lieu) et la hauteur moyenne (mieux liée au volume sur pied), et c'est la hauteur moyenne des arbres dominants et co-dominants qui a été utilisée dans l'étude. Celle-ci coïncide en général avec la hauteur moyenne dont elle ne s'écarte que dans des cas exceptionnels.

La croissance en hauteur d'un peuplement peut être étudiéc de la meilleure façon par des analyses de tiges dont nous ne pouvions disposer, et le travail effectué a consisté à conduire cette étude à partir de mesures provenant de différents peuplements une seule fois mesurés. Cette tentative n'est pas nouvelle et nous verrons les difficultés qu'elle présente, de même que la façon suivant laquelle nous avons proposé de les résoudre. Pour cela nous pouvions compter à la fois sur les mesures dendrométriques et sur les mesures des conditions de milieu des placettes de l'Inventaire Forestier National. Sur ces conditions étaient connues avec une précision variable : laltitude, la pente, l'exposition, et des appréciations sur la profondeur du sol, sa composition, de même que lassise géologique. Facteurs qui sont autant de sources de variabilité de la croissance des peuplements, et dont nous nous sommes efforcés, avec des fortunes diverses, d'apprécier les effets. Cest laltitude, le plus important de ces facteurs, qui a pu être contrôlée, avec comme but de rendre plus précise la relation entre lâge et la hauteur des placettes, de façon à procurer des courbes de croissance plus fidèles.

\section{II. - Présentation de la région d'étude}

\section{1. - Limites géographiques}

Au sens large du terme, la Margeride couvre trois fractions de département : Lozère, Haute-Loire, et Cantal. Ses limites sont à l'est la vallée de l'Allier, à l'ouest celle de la Truyère, au sud les Causses de Mende et de Montbel, et au nord les terrains volcaniques (voir O. NOUgaride, 1977). Cette région naturelle est bien incluse dans la région définie par linventaire Forestier National (I.F.N.) portant le mème nom. Nous nous sommes cependant limités aux stations de Pin sylvestre situés sur granite, car les travaux de l'ensemble du groupe d'étude des systèmes agro-sylvopastoraux en Margeride (laction D.G.R.S.T. que nous avons déjà citée) se situait dans les limites du faciès granitique, en gros au milieu du triangle délimité par les villes de Saint-Flour, le Puys et Mende. Les sols développés sur les matériaux volcaniques ayant, en général, une potentialité forestière plus élevée que celle des 
sols développés sur granite (LE TACON et al., 1970), cette restriction avait donc de plus un sens du point de vue des questions qui nous concernaient, en établissant un ensemble plus homogène.

\section{2. - Caractéristiques du milieu physique}

La Margeride granitique est composée principalement de deux zones : les plateaux étagés entre $700 \mathrm{~m}$ et $1100 \mathrm{~m}$, coupés de vallées parfois encaissées, et la montagne de la Margeride proprement dite qui est une ligne de crêtes culminant vers $1500 \mathrm{~m}$. C'est un massif ancien granitique composé de leucogranite avec des roches filoniennes (microgranites, quartz).

Le climat de cette région a bien été décrit par Daget (1967). Le climat est de type montagnard aux hivers froids et longs. Il subit les influences océaniques, continentales, et méditerranéennes. Les variations locales sont importantes du fait du relief. La pluviométrie annuelle est d'environ $900 \mathrm{~mm}$ sur les plateaux, de $1100 \mathrm{~mm}$ sur les crêtes. Les températures moyennes varient de 5 à $8^{\circ}$. La saison de végétation est assez courte : de 140 jours en altitude à 180 jours sur les plateaux.

Les sols développés sur ce granite leucocrate ont un certain nombre de caractéristiques communes : ce sont des sols acides, la texture sableuse est dominante, la profondeur du sol et la teneur en cailloux des profils sont liées à la topographie. Ces sols sont chimiquement pauvres en bases échangeables et désaturés. Des observations faites en parcourant la Margeride confirment linfluence de l'altitude sur la pédogenèse : aux a!titudes inférieures existent principalement des sols bruns acides. Plus l'altitude augmente, et plus la pédogenèse podzolique est accentuée. Sur les crêtes se situent les sols de type ranker-cryptopodzolique. Cette répartition est confirmée par les travaux de Dupuis \& Horemans (1966), de Long \& Daget (1965), en Margeride, et dans les régions voisines telles l'Ardèche (LE Tacon, Oswal.d, 1969) et le plateau de Millevaches (Nys, 1973).

\section{3. - La végétation}

En résumé et d’après Daget (1967) puis Barbet (1978), deux zones de végétation peuvent grossièrement être distinguées. En altitude, à plus de $1400 \mathrm{~m}$ où le sol est de type ranker-cryptopodzolique, la végétation caractéristique est la lande à callune. La zone des plateaux est l'étage de la hêtraie. Les bas-fonds hydromorphes sont occupés par des tourbières.

Le pin sylvestre : en Margeride granitique le Pin sylvestre sous ses différents types de peuplement occupe 70000 hectares représentant à peu près le quart de la région considérée. C'est rarement une espèce de reboisement mais plus souvent une espèce de colonisation des espaces abandonnés par l'agriculture (DAGET, 1967 ; BARBET, 1978 ; Nougarede, 1977). Les peuplement pleins de pins sylvestres se situent dans les zones forestières soumises ou protégées, rarement en terrains privés. Le plus souvent cette espèce constitue des près-bois ou des pacages-bois. 


\section{III. - Les données dendrométriques de l'I.F.N. et la conduite des premières actions}

L'unité d'échantillonnage au sol de l'Inventaire Forestier National est constituée par trois placettes circulaires concentriques de $6 \mathrm{~m}, 9 \mathrm{~m}$, et $15 \mathrm{~m}$ de rayon. Sur chaque unité d'échantillonnage les arbres sont pris en compte et mesurés de la façon suivante : sur la plus petite placette, les arbres dont le diamètre est compris entre $7,5 \mathrm{~cm}$ et $22,5 \mathrm{~cm}$; sur la placette intermédiaire, les arbres de diamètre compris entre $22,5 \mathrm{~cm}$ et $37,5 \mathrm{~cm}$, et sur la plus grande, les arbres dont le diamètre est supérieur à $37,5 \mathrm{~cm}$ (1) (cette distinction est probablement destinéc à équilibrer les précisions relatives des variables de peuplement estimées à l'hectare pour les arbres de diverses dimensions). Sur chaque arbre retenu sont mesurées les grandeurs suivantes : le diamètre à $1,30 \mathrm{~m}$, et à ce niveau l'épaisseur d'écorce (avec la jauge à écorce), ainsi que la largeur des cing derniers cernes (avec la tarière de Pressler); la hauteur totale de l'arbre est mesurée à la perche ou au dendromètre de Christen. De plus, sur chaque unité d'échantillonnage deux arbres sont sondés jusqu’au cour à leur pied pour en déterminer l'âge. Désormais, nous appellerons simplement «placette » cette unité d'échantillonnage composée.

Parmi l'ensemble des placettes de Pin sylvestre de la région considérée, un premier tri a écarté celles dont les deux arbres sondés au cour présentaient une différence d’âge supérieure à 5 ans, de façon à réduire l'imprécision sur l'âge estimé par la moyenne de ces deux valeurs : il s'agissait aussi d'augmenter les chances de travailler avec des placettes équiennes (2). C'est cette valeur moyenne qui sera l' «âge» des placettes. La hauteur moyenne des arbres dominants et co-dominants a été calculée (en tenant compte des particularités de l'échantillonnage qui viennent d'être décrites) pour les placettes restantes (environ 300) grâce aux notes de I'I.F.N. qui indiquaient les arbres dominés; cette valeur moyenne sera simplement appelée " hauteur» dans ce qui suit.

Ayant été sensibilisés à la possibilité d’être en présence de différents types de pins sylvestres à cause de différentes provenances ou des effets de mutilations précoces dues au bétail (voir BARBET, 1978, pour cette discussion), il fut tenté de distinguer parmi les placettes différentes «populations » en examinant les coefficients de forme des arbres, ou plus simplement les diamètres des tiges à la découpe (1); mais la régularité de la distribution de ces valeurs ne permettait pas de faire de distinction : aucun nouveau tri ne put être justifié sur cette base. Cependant, chacune des placettes a finalement été examinée et une vingtaine d'entre elles ont été écartées pour des raisons particulières, mais généralement parce qu'elles comptaient moins de 4 arbres ou parce qu'elles avaient une trop grande proportion d'arbres mal formés (ayant une fourche très basse, ou un diamètre à la découpe trop éloigné du diamètre normal $\mathrm{de} 7 \mathrm{~cm}$ ). Il restait 277 placettes pour poursuivre l'étude.

(1) Ces limites correspondent aux catégories de petits bois, bois moyens, et gros bois classiquement utilisés par les forestiers (cf. Manuel d'Aménagement de 1'O.N.F., 2é édition, p. 78).

(2) Ce qui est d'autant plus vraisemblable, pour des placettes suffisamment denses, que le $P$ in sylvestre est une essence de lumière.

(1) I.e diamètre à la découpe est normalement de $7 \mathrm{~cm}$; lorsqu'un arbre présente des malformations graves il est ramené au diamètre de la tige au-dessous du défaut. 


\section{IV. - Principes d'étude et analyse des données}

Nous rappelons que le but du travail exposé ici était d'obtenir des courbes caractéristiques de la croissance en hauteur de peuplements de pins sylvestres pour des stations de différentes productivités, à partir des mesures provenant de placettes temporaires. Cette opération est généralement menée en utilisant la méthode dite "de Bruce \& Schumacher» (1950) qui consiste à déduire des données sur lâge et la hauteur des placettes une courbe caractéristique de la croissance en hauteur, doù s'obtiennent les courbes particulières à chaque classe de productivité au moyen d'une transformation simple. Cette transformation est telle que le rapport des hauteurs indiquées par deux quelconques de ces courbes à un âge donné soit indépendant de l'âge (affinité). Ce principe de courbes affines pour représenter la croissance en hauteur des peuplements dans les différentes stations a parfois été mis en défaut par des études conduites sur des courbes de croissance en hautcur provenant d'analyses de tiges (KING, 1966 et Decour'r, 1972, ont indirectement fait cela, mais cette démonstration a aussi été donnée par des études menées dans ce but). Des travaux effectués dans ce domaine il se dégage cependant lidée que le principe des courbes affines dont rous venons de parler est souvent vérifié, et qüil donne une description approximative des phénomènes qui est satisfaisante dans la majorité des cas. Quoi quil en soit, il est bien difficile de ne pas l'utiliser lorsque l'on ne dispose pas danalyse de tige. Par contre nous nous sommes efforcés de remédier à un autre défaut de la méthode de Bruce \& Schumacher que Decourt a signalé bien que layant lui-même utilisée (Decourt, 1965) : les conditions de milieu ont des effets importants sur la croissance en hauteur; les courbes figurant la croissance en hauteur obtenues par cette méthode peuvent en partie refléter cela de façon indésirable, et donner des estimations biaisées, c'est-à-dire se trouver au-dessus ou au-dessous des valeurs qu'elles devraient avoir à un âge donné. Tenir compte explicitement de ces effets lorsque cela est possible permet de réduire ces biais. C"est ce que nous avons cherchá à faire et le paragraphe suivant expose lapproche suivie.

Lanalyse des données expérimentales est considérablement facilitée par une connaissance générale des phénomènes que peuvent procurer les travaux antérieurs. Ainsi pour savoir quel peut être l'effet de lexposition et de la pente d'un lieu considéré sur la productivité (autrement dit sur la croissance en hauteur), les articles de Myers \& Deusden (1960), Beer et al. (1966) et de Stage (1976), entre autres, sont précieux. Mais nous avons été particulièrement sensibilisés aux importants effets cie laltitude sur la croissance dans les régions de moyenne montagne par les articles de OSwal.D (1969) et Decourt \& NYs (1976). C'est cet effet que nous avons d'abord cherché à mettre en évidence et à contrôler. Pour éviter les discontinuités pouvant être introduites par des conditions topographiques trop particulières, les placettes situées dans les fonds de vallons, ou au contraire sur des crêtes furent écartées. La figure 1 présente les couples de valeurs des âges et des hautcurs pour les 246 placettes restantes : on y discerne lallure caractéristique de la croissance en hauteur. Il est plus difficile de saisir de la même façon les rapports entre l'altitude et la hauteur des placettes car celle-ci est déjà fortement déterminée par l'âge et cet effet masque celui de l'altitude. Une courbe «moyenne» de croissance cn hauteur ajustée avec une certaine subjectivité (de façon informelle) à partir des données de la figure 1 peut servir à représenter provisoirement l'effet de l'âge sur la hauteur. Il devient 
alors possible d'étudier si l'altitude rend compte de la façon dont une valeur observée de la hautcur à un âge donné s’écarte ce la courbe moyenne. Cet écart peut être mesuré par le rapport de la valeur observée à la hauteur moyenne indiquée par la courbe en question à laâge correspondant (il représente alors d'une certaine façon la productivité du lieu où se trouve la placette considérée). L'examen simultané des rapports ainsi calculés et des altitudes a permis d'observer le schéma de dépendance suivant : jusquà laltitude de $1000 \mathrm{~m}$ environ il n'y a pas d'influence sensible de l'altitude sur la croissance en hauteur; un peu au-dessus de $1000 \mathrm{~m}$ la productivité décroît linéairement en fonction de l'altitude. Cette forme de dépendance a déjà été observéc par Decourt \& Nys (1976) avec le douglas, et ce précédent a permis d’identifier assez facilement le phénomène. Le graphique ayant servi à cette analyse n'est pas reproduit ici, mais la figure 3 qui apparaîtra comme le résultat du traitement numérique des données que nous venons de décrire, permettra, d'une certaine façon, d'appréhender cet effet.

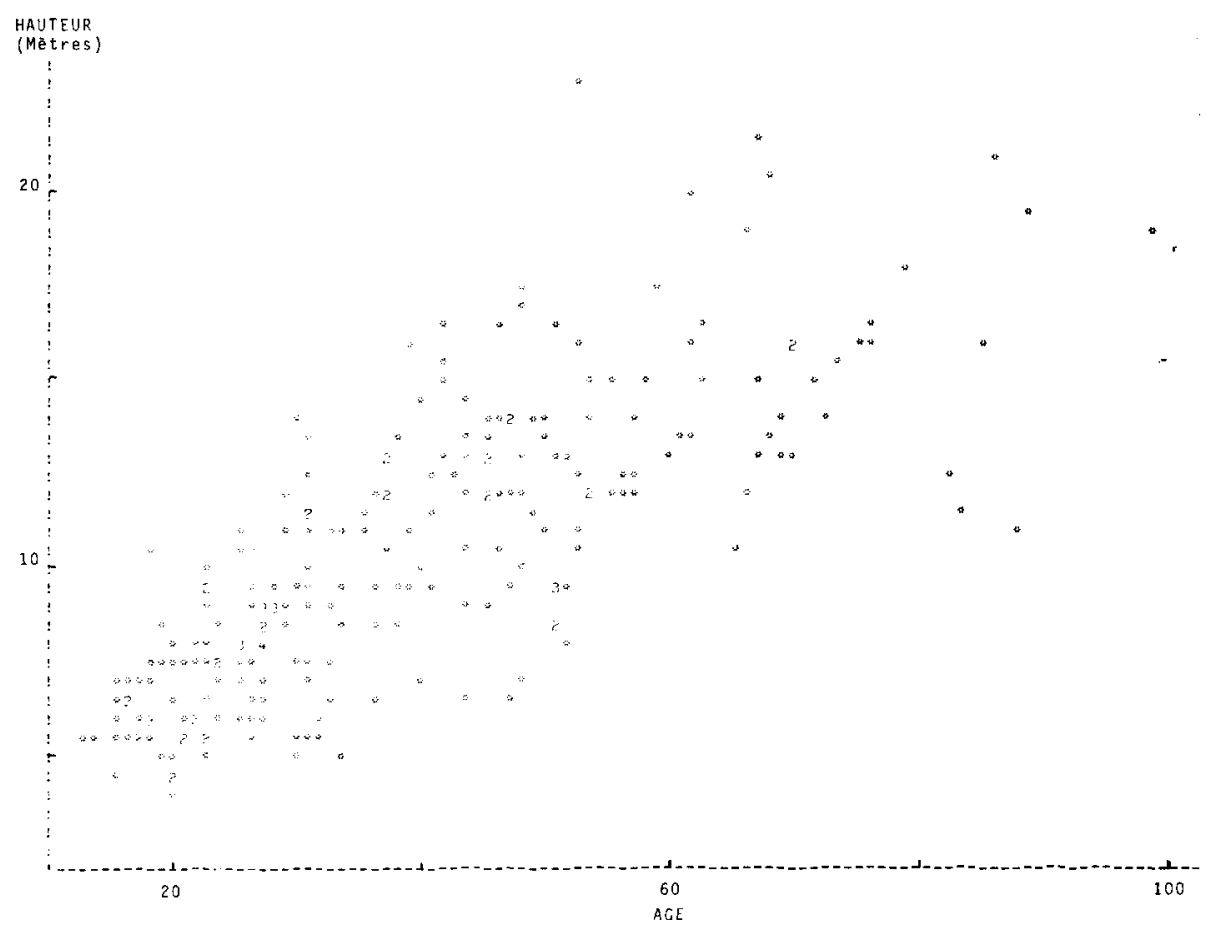

Figure 1

Age et hautcur moyenne des arbres dominants ei codominants des placentes ciudices: distribution des hauteurs suivant l'âge

Scatter plot of the dominant and co-dominant trees mean height against age (the former variable will be simply referred as the «height in the subsequent figures) 


\section{V. - Traitement et exploitation des données}

Il restait, à ce stade, à tenir compte des principaux phénomènes reconnus pour atteindre le but fixé. Si lon postule que la hauteur HCD à un âge donné $\mathrm{A}$, et dans les conditions de station I, est (à un facteur aléatoire près) de la forme :

$$
\mathrm{HCD}=\mathrm{f}(\mathrm{A}) \cdot \mathrm{g}(\mathrm{I})
$$

où $\mathrm{f}$ est une fonction de croissance ne dépendant que de l'âge, $g$ est une fonction des conditions de milieu qui représente leurs effets sur la croissance, on se trouve en accord avec le principe des courbes affines dont nous avons parlé (ce principe a déjà été formulé de la même façon par Decourt \& Lemorne, 1969). Pour être aussi en accord avec l'analyse des phénomènes qui vient d'être évoquée, il suffit de donner à l'altitude une forme adéquate : l'altitude critique que nous avons signalée a été fixée à $1040 \mathrm{~m}$ (cette valeur était suggérée par la représentation graphique des données analysées et l'estimation formelle de ce paramètre impliquait une difficulté technique dont la charge semblait injustifiée), et $\mathrm{g}$ a été paramétrée sous la forme dune fonction linéaire par morceaux qui est constante jusquà $1040 \mathrm{~m}$ et peut prendre une pente à partir de cette valeur de l'altitude. Ainsi, la forme complète de la hauteur exprimée comme fonction de l'âge et de l'altitude s'écrit :

$$
\begin{aligned}
& \mathrm{HCD}=\mathrm{K}_{1}\left(1-\mathrm{e}^{-\mathrm{K}^{2} \mathrm{~A}}\right) \text { pour } \mathrm{ALT}<1040 \\
& \mathrm{HCD}=\mathrm{K}_{1}\left(1-\mathrm{e}^{-\mathrm{K}^{2} \mathrm{~A}}\right)\left\{1+\mathrm{K}_{3}(\mathrm{ALT}-1040)\right\} \text { pour } \mathrm{ALT} \geqslant 1040
\end{aligned}
$$

(la fonction de croissance $f$ est une forme simplifiée de la fonction Richards-BertaI.ANFFY car au cours du calcul des estimations la présence du paramètre supplémentaire s'est avérée inutile). L'estimation des paramètres $\mathrm{K}_{1}, \mathrm{~K}_{2,}$ et $\mathrm{K}_{3}$ fut obtenue comme en régression linéaire en déterminant les valeurs minimisant l'expression $S^{2}\left(K_{1}, K_{2}, K_{3}\right)$ telle que :

$$
\mathrm{S}^{2}\left(\mathrm{~K}_{1}, \mathrm{~K}_{2,}, \mathrm{~K}_{3}\right)=\sum_{\mathrm{i}=1}^{\mathrm{n}}\left\{\mathrm{HCD}_{\mathrm{i}}-\mathrm{f}\left(\mathrm{A}_{\mathrm{i}}\right) \mathrm{g}\left(\mathrm{ALT}_{\mathrm{i}}\right)\right\}^{2}
$$

où $n$ est le nombre des observations (nombre de placettes), $\mathrm{HCD}_{\mathrm{i}}$ la hauteur de la placette $\mathrm{i}$ d'âge $A_{i}$ à l'altitude $A_{L} T_{i}$.

Draper et SMith (1966) recommandent comme une bonne pratique de procéder après une estimation à l'examen simultané des valeurs résiduelles et des valeurs estimées. Ici les valeurs résiduelles sont les différences $\left(\mathrm{HCD}_{\mathrm{i}}-\mathrm{HCD}_{\mathrm{i}}\right)$ où $\mathbf{H C D _ { i }}$ est la valeur estimée de $\mathrm{HCD}_{\mathrm{i}}$ calculée en remplaçant les paramètres $\mathrm{K}_{1}, \mathrm{~K}_{3}, \mathrm{~K}_{3}$, qui apparaissent dans $\mathrm{f}$ et $\mathrm{g}$, par leurs valeurs estimées. L'examen de la figure 2 où ces valeurs sont représentées montre que la dispersion des valeurs résiduelles augmente avec la valeur estimée. La méthode d'estimation décrite ci-dessus n'étant pas prévue pour tenir compte de ce phénomène, les résultats obtenus dans ces cas sont généralement médiocres. De fait, il apparâit dans la figure $(2$ a) une surestimation des faibles valeurs de la hauteur qui se traduit près de lorigine des abscisses par un trop grand nombre de valeurs négatives des différences $\left(\mathrm{HCD}_{\mathrm{i}}-\mathrm{HCD}_{\mathrm{i}}\right)$; par contre les valeurs moyennes de la hauteur (entre $10 \mathrm{~m}$ et $15 \mathrm{~m}$ ) sont sous-estimées. Ce défaut a été confirmé par l'examen des valeurs résiduelles $\left(\mathrm{HCD}_{\mathrm{i}}-\mathrm{HCD}_{\mathrm{i}}\right)$ avec les variables 


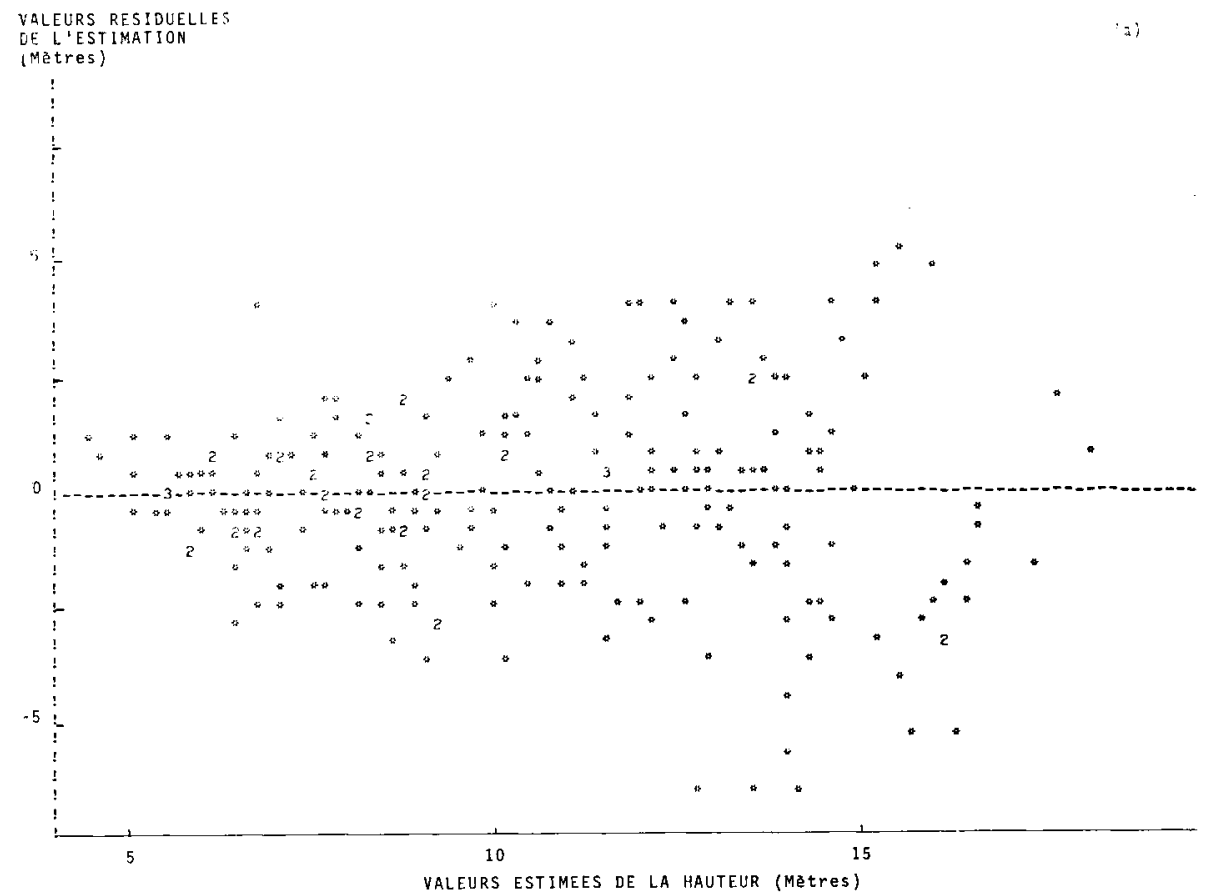

VALEURS RESIOUELLES PONOEREES

DE L'EST IMAT ION

(b)

(Unités non definies)

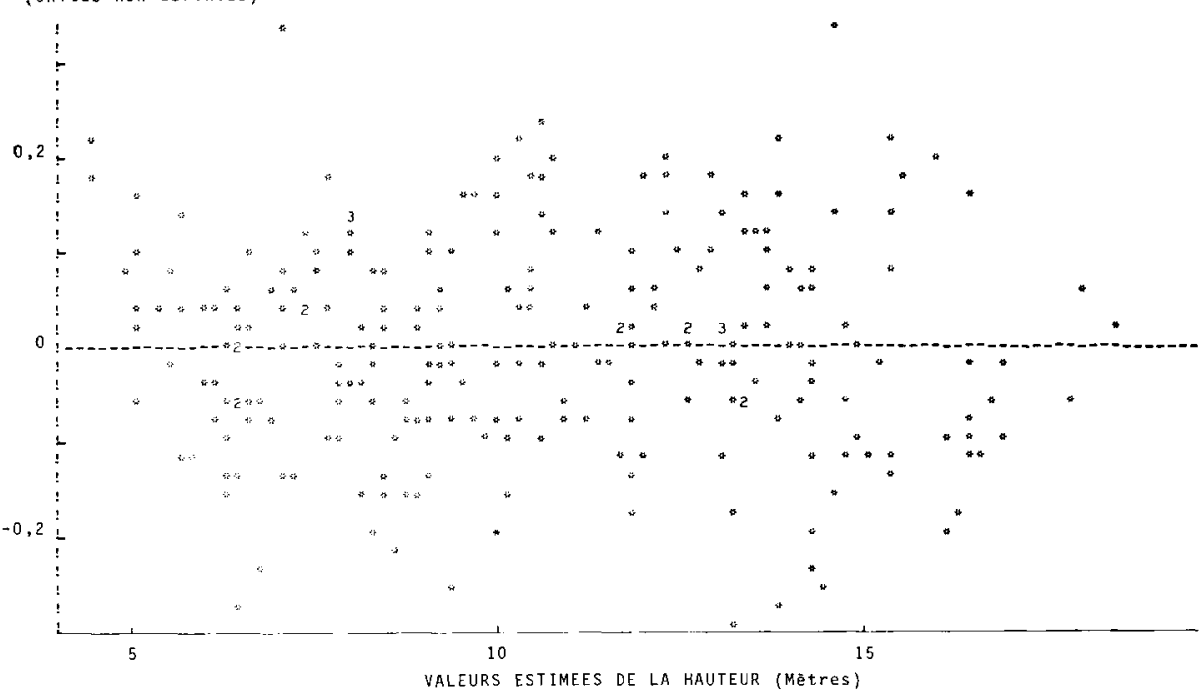

Figure 2

Dispersion, suivant la valeur estimée,

des résidus issus de l'estimation de la hauteur des placettes par une fonction de l'âge et de l'allitude : (a) un procédé supposant l'homogénéité de la dispersion a été utilisé ;

(b) le même procédé a été modifié pour tenir compte de l'accroissement de la dispersion avec la valeur estimée

Scatter plot of the residual differences between the observed values of the stands height and those estimated by a function of elevation and age :

(a) using regular non-linear regression;

(b) using the same process with iteratively estimated weights 
explicatives (âge et altitude). Afin de rendre son efficacité à la méthode d"estimation, une version très simplifiée de la procédure de Box \& HıL. (1974) a été appliquée : moyennant certaines précautions, la première estimation obtenuc a seulement servi à estimer les pondérations nécessaires pour corriger l'augmentation de la dispersion qui apparaît à la figure 2 (a) dans des applications ultéricures de la méthode classique d'estimation. Le résultat final de ces opérations apparaît à la figure 2 (b) où la dispersion des valeurs résiduelles (qui sont portées en ordonnée), corrigée progressivement au cours du processus destimation, apparaît davantage homogène du fait qu'elle n`augmente plus avec les valeurs estimées (en abscisse). L'examen de ces valeurs conjointement à celles des variables explicatives ne permettait pas de déceler de biais dans les estimations. L'expression finale de la hauteur des placettes en fonction de l'âge et de l'altitude (conformément à ce qui précède) est donnée ci-dessous.

$$
\left\{\begin{array}{cl}
\mathrm{HCD}=20,74\left(1-\mathrm{e}^{-(0,02: 30+\Lambda)}\right. & \text { pour } \mathrm{ALT}<1040 \\
\mathrm{HCD}=20,74\left(1-\mathrm{e}^{-0.0,12: 304 \Lambda}\right) & \{1-0,001012(\mathrm{ALT}-1040)\} \\
& \text { pour } \mathrm{ALT} \geqslant 1040
\end{array}\right.
$$

avec donc les estimations :

$$
\begin{aligned}
& \widehat{\mathrm{K}}_{1}=20,74 \\
& \widehat{\mathrm{K}}_{2}=0,02304 \\
& \widehat{\mathrm{K}}_{3}=-0,001012
\end{aligned}
$$

les unités étant :

- la hauteur et laltitude des placettes en mètres,

- I’âge en années.

L'estimation cherchée ayant été obtenue, il est alors intéressant de faire la remarque suivante : les valeurs trouvées sont celles qui minimisent l'expression $\mathrm{S}^{\prime 2}\left(\mathrm{~K}_{1}, \mathrm{~K}_{\underline{.},}, \mathrm{K}_{3}\right)$ telle que :

$$
\mathrm{S}^{\prime 2}\left(\mathrm{~K}_{1}, \mathrm{~K}_{2}, \mathrm{~K}_{3}\right)=\sum_{\mathrm{i}=1}^{\mathrm{n}} \mathrm{W}_{\mathrm{i}}\left\{\mathrm{HCD}_{\mathrm{i}}-\mathrm{f}\left(\mathrm{A}_{\mathrm{i}}\right) \mathrm{g}\left(\mathrm{ALT}_{\mathrm{i}}\right)\right\}
$$

où les $W_{\mathrm{i}}$ sont des pondérations qui ont été obtenues au cours du processus d'estimation. Dès lors, $\widehat{\mathbf{K}}_{3}$ peut apparaître comme la valeur de $\mathbf{K}_{3}$ qui rend minimum l'expression $S^{\prime 2}\left(K_{3}\right)$ définie en remplaçant $K_{1}$ et $K_{2}$ dans la relation (5) par leurs valeurs estimées. Pour préciser cela, posons :

$$
\begin{aligned}
& \mathrm{y}_{\mathrm{i}}=\mathrm{HCD}_{\mathrm{i}}-20,74\left(1-\mathrm{e}^{\left.-0,02304 \mathrm{~A}_{\mathrm{i}}\right)}\right. \\
& \mathrm{x}_{\mathrm{i}}=0 \text { si } \operatorname{ALT}_{\mathrm{i}}<1040 \\
& \mathrm{x}_{\mathrm{i}}=20,74\left(\mathrm{~A}-\mathrm{e}^{-0,0230+\Lambda_{\mathrm{i}}}\right)\left(\mathrm{ALT}_{\mathrm{i}}-1040\right) \text { si } \mathrm{ALT}_{\mathrm{i}}>1040
\end{aligned}
$$

L'expression $\mathrm{S}^{\prime 2}\left(\mathrm{~K}_{3}\right)$ s'écrit simplement :

$$
\mathrm{S}^{\prime 2}\left(\mathrm{~K}_{3}\right)=\sum_{\mathrm{i}=1}^{\mathrm{n}} \mathrm{W}_{\mathrm{i}}\left\{\mathrm{y}_{\mathrm{i}}-\mathrm{K}_{3} \mathrm{x}_{\mathrm{i}}\right\}^{2}
$$




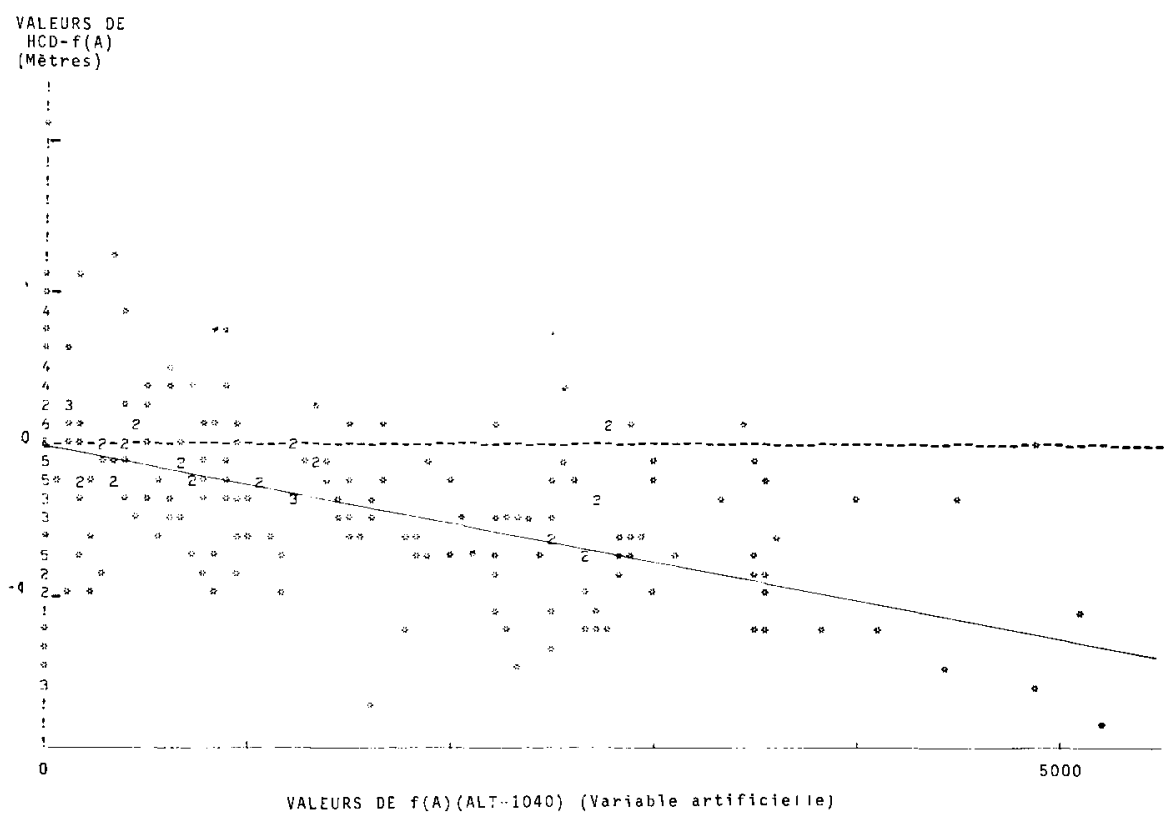

FigURE 3

Représentation graphique (par la pente de la droite de régression) d'un paramètre estimé, destinée à mettre en évidence la contribution de l'altitude dans l'estimation évoquée à la figure (2) (équation (4) dans le texte)

Graph intended to attest for the effect of elevation in the estimation of the stands height given by equation (4) : the slope of the simple regression line equals the estimated parameter standing for this effect

De cette façon $\widehat{\mathrm{K}}_{3}$ se présente comme la pente d'une droite de régression qui lie les valeurs $y_{i}$ aux valeurs $x_{1}$. On peut constater cela à la figure 3 où cette droite a été tracée et les valeurs des variables artificielles $x_{i}$ et $y_{i}$ portées en abscisse et en ordonnée. La procédure qui vient d'être décrite est une adaptation de celle développée par LARSEN \& MCLEARY pour la régression linéaire.

Sans chercher à donner un sens aux variables $x_{i}$ et $y_{i}$, l'intérêt de cette remarque est le suivant : il permet d'apprécier la contribution de l'altitude dans les relations (4) : les couples $\left(x_{i}, y_{i}\right)$ se dispersent autour de droite de pente $\widehat{K}_{3}=-0,001012$ qui s'écarte sensiblement de l'axe des abscisses, compte tenu de la dispersion moyenne des points. Apparemment, les pondérations $W_{i}$ n'interviennent que pour compenser les variations de la dispersion, conformément au principe suivant lequel elles ont été calculées. Par ailleurs, $\widehat{K}_{: ;}$est supposé rendre compte de l'influence de l'altitude sur la croissance, mais on peut se demander s'il ne reflète pas en partie un effet de l'âge (comme cela se serait passé s'il y avait eu une liaison statistique entre l'âge 
des placettes et l'altitude, chose qui n'a pas été constatée); il semble que l'on puisse écarter cette possibilité car à l'examen la variable $\mathrm{x}_{\mathrm{i}}$ ne présentait aucune corrélation avec l'âge.

\section{VI. - Application}

Il fut tenté vainement de pousser plus loin l'analyse qui vient d'être présentée pour l'étendre aux autres facteurs susceptibles d'avoir des effets sur la productivité : exposition, pentes, profondeurs de sol, à partir des informations qui étaient disponibles sur ces diverses conditions pour chaque placette. L'une des raisons pour lesquelles l'analyse de l'influence de ces autres facteurs du milieu sur la croissance en hauteur n'a pu donner de résultat est très probablement liée au fait que, excepté pour les pentes, les valeurs par lesquelles ces facteurs se trouvaient représentés étaient groupées en classes. Ce manque de précision dans leur évaluation introduisait visiblement une variabilité irréductible très forte rendant de même impossible à détecter une relation entre les facteurs en question et l'altitude avec laquelle, par ailleurs, les pentes ne se trouvaient pas correlées.

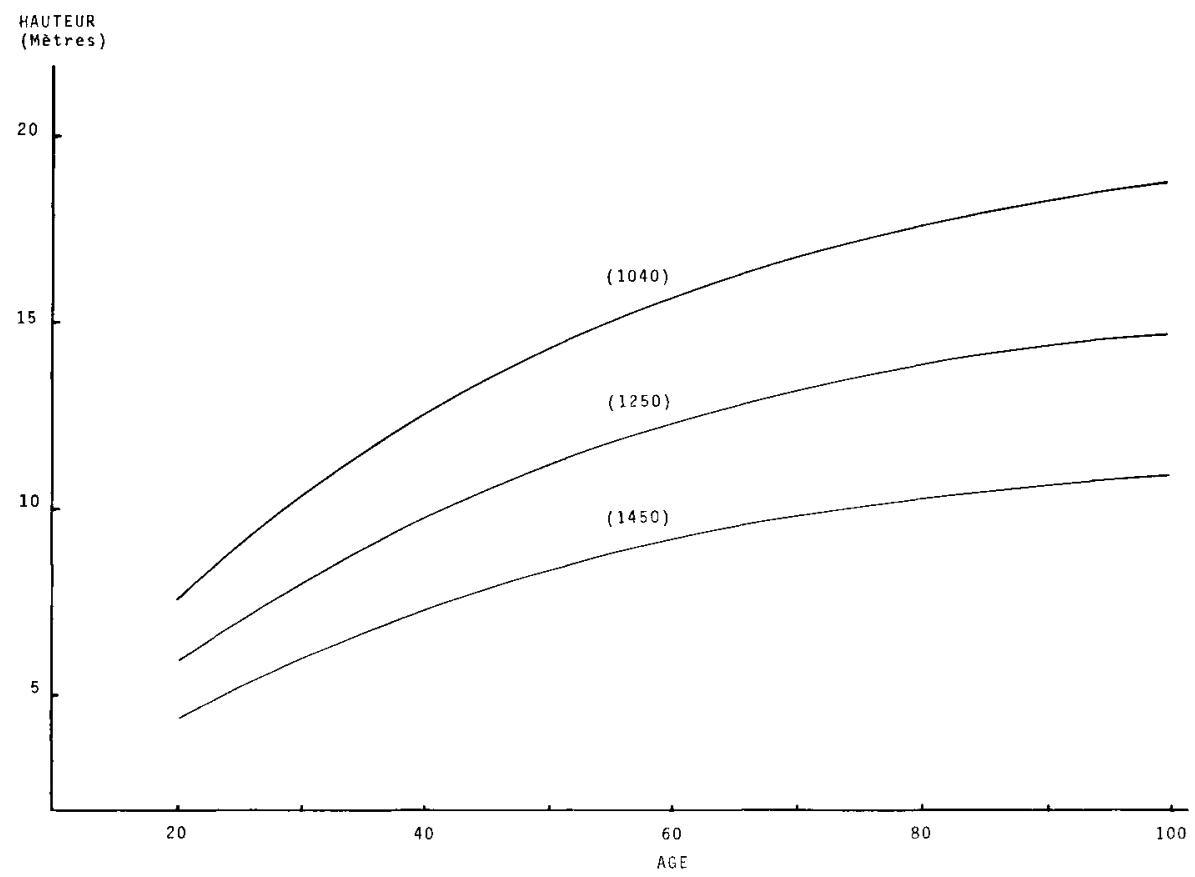

Figure 4

Courbes schématisant la croissance en hauteur à différentes altitudes: jusqu'à $1040 \mathrm{~m}$, à $1250 \mathrm{~m}$, à $1450 \mathrm{~m}$ (abstraction est faite de la variabilité)

Characteristic height-age curves for different elevations 
Pourtant, de la possibilité d'avoir pu faire la part des effets de l'altitude sur la hauteur observée des placettes il est attendu de la représentation des courbes de croissance en hauteur du Pin sylvestre en Margeride qu'elle soit plus fiable qu'elle ne l'aurait été si' l'on n'avait pas tenu compte de cet effet. La figure 4 illustre cette application : le comportement typique de la croissance en hauteur y est représenté pour les altitudes de $1040 \mathrm{~m}$ (et au-dessous), $1250 \mathrm{~m}$, et $1450 \mathrm{~m}$. Il semble en superposant les figures 1 et 4 que les hauteurs (à un âge donné) soient sous-estimées pour les meilleures stations, mais cela provient du fait que la dispersion des valeurs de la hauteur a tendance à augmenter avec la productivité. A cause de cette dispersion assez importante on ne peut accorder aux altitudes de référence qui viennent d'être citées qu'une valeur indicative n'ayant réellement de sens que pour un grand nombre de cas en moyenne. Ainsi il faut savoir que même au-dessous de $1040 \mathrm{~m}$ on peut trouver des stations de très faible productivité, à cause des sources de variabilité qui n'ont pu être contrôlées dans cette étude. Cependant, la figure 5 donne une représentation schématique de l'effet de l'altitude sur la productivité (identifiée à la hauteur à 50 ans) tel qu'il se présente donc en moyenne. Utilisé avec prudence, ce schéma peut rendre certains services.

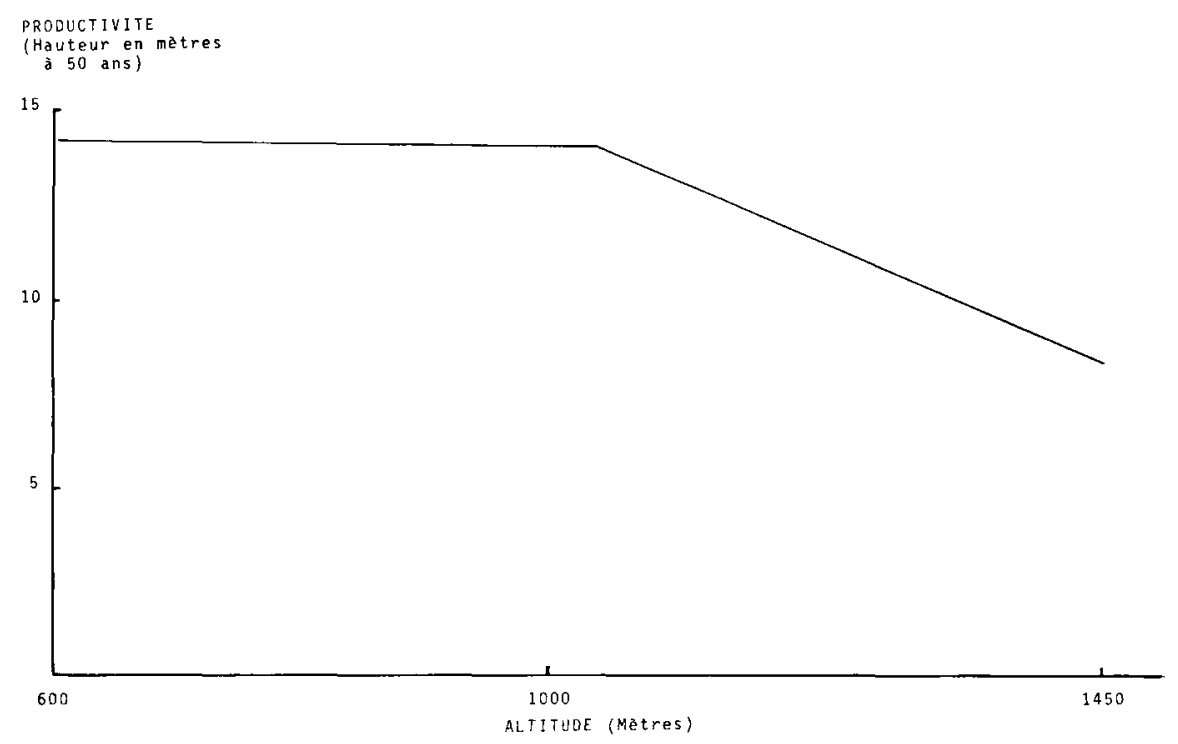

Figure 5

Variation de la productivité moyenne avec l'altitude

Varying mean productivity versus elevation

\section{VII. - Conclusion}

En partant de données initialement recueillies dans un intervalle de temps limité pour les besoins de l'inventaire forestier d'une grande étendue, un traitement particulier permet d'en déduire l'évolution caractéristique de la hauteur d'un peuplement de 
productivité donnée au cours du temps. On peut de plus noter que dans les conditions où cette estimation a été réalisée, des indications ont pu être obtenues sur la façon dont la productivité des stations varie avec la plus marquante des conditions physiques du lieu dans la région considérée : l'altitude.

Ces résultats acquis, après avoir vérifié qu’ils ne présentent pas de vices plus ou moins grossiers et détectables dans le contexte où ils sont apparus, on peut vouloir pousser plus loin le désir de les mettre à l'épreuve. Cela n’est possible de façon vraiment sûre que si l'on dispose de données provenant de placettes permanentes qui ont été suivies sur un temps assez long, ou si l'on peut procéder à quelques analyses de tiges. Cette vérification sort du cadre de la présente étude, mais pourrait être conduite sans trop de difficultés si le besoin s'en présentait : et par exemple à l'occasion de l'extension de la méthode qui a été présentée à d'autres essences, ce contrôle pourrait être un préliminaire souhaitable.

Beaucoup de soins ont été consacrés à l'étude de la croissance en hauteur du Pin sylvestre en Margeride car elles constituent un élément essentiel permettant de déterminer sa production totale en volume. Cette étude de la production totale a effectivement été conduite sur la base des courbes de croissance en hauteur obtenues; ces travaux seront le propos d'un autre article.

Reçu pour publication en janvier 1980.

\section{Summary}

Growth and yield of Scot pine in Margeride using data from I.F.N.

\section{A. - The height growth}

The yield of Scot pine stands of an agricultural region in the central part of France (Margeride) had to be investigated for the needs of a socio-economic study. In order to avoid the load of new field measurements, the data available from a survey of the national organization taking charge of the forests inventory (I.F.N.) were used. The present paper reports the first part of the study in which the site curves giving the characteristic heightage relationships for the sites of different productivities were estimated. As a main result, the height of temporary sample plots could have been directly related to their age and elevation using non-linear regression. Technical difficulties came up with unhomogeneous unexplained variability of the response. To deal with these difficulties, practical solutions of intuitive meaning were proposed, in connection to simpler and well solved problems.

\section{Références bibliographiques}

Barbet C., 1978. Le Pin sylvestre en Margeride. Mémoire de 3" année E.N.I.T.E.F., 45 Nogent-sur-Vernisson.

Beers T.W., Dress P.E. Whensel L.C., 1966. Aspect transformation in site productivity research. J. For., 64, 691-692. 
Box G.E.P., Hill W.J., 1974. Correcting inhomogeneity of variance with power transformation weighting. Technometrics, 16 (3), 385-389.

Bruce O., Schumacher F., 1950. Forest Mensuration. McGraw Hill Book Company, New York, 1950.

DAGET P., 1967. Etude phyto-climatique d'une région de moyenne montagne : La Margeride. Doc. $\mathrm{n}^{\circ}$ 36, C.N.R.S., C.E.P.E., B.P. 1018 Montpellier.

Dupuis J., Horemans P., 1966. Observations sur quelques sols du Massif Central. Ann. Inst. natl. agron., VI, 317-375.

Décourt N., 1965. Le Pin sylvestre et le Pin laricio de Corse en Sologne. Tables de production provisoires et méthode utilisée pour les construire. Ann. Sci. for., 22 (2), 259-318.

Décourt N., 1972. Méthode utilisée pour la construction rapide de tables de production provisoires en France. Ann. Sci. for., 29 (1), 35-48.

Décourt N., Lemorne B., 1969. Le Pin maritime dans le sud-ouest de la France. Tables de production provisoires. $A n n . S c i$. for., 26 (1), 3-44.

Décourt N., Nys C., 1976. Nutrition, croissance et production de l'Epicéa et du Douglas dans le Limousin. Rev. for. fr., XXVIII (3), 193-200.

Draper N.R., Smith H., 1966. Applied regression analysis. John Wiley and Sons, New York.

KING J., 1966. Site index curves for Douglas-fir in the Pacific Northwest. Weyerhaeuser Forestry, Paper $\mathrm{n}^{\circ} 8$.

Larsen W.A., McLeary S.J., 1972. The use of partial residual plots in regression analysis. Technometrics, 14 (3), 781-790.

Le Tacon F., Oswald H. 1969. Les sols de Haute Ardèche : caractérisation et production forestière. Sci. Sol, A.F.E.E., $\mathrm{n}^{\circ} 2$.

LE Tacon F., Oswald H., Tomassone R., 1970. La nutrition minérale de l'Epicéa en Haute Ardèche. Liaison avec la production. Ann. Sci. for., 27 (4), 357-381.

Long G., Daget P., 1965. Contribution à l'étude écologique du massif de la Margeride. Observations sur la pédogénèse et le dynamisme de la végétation sur les granites. Ann. agron., $16(4), 401-432$.

Myers C.A., Van Deusden J.L., 1960. Site index of ponderosa pine in the Black Hills from soil and topography. J. For., 58, 548-555.

Nougarede O., 1977. Premières réflexions concernant les équilibres agro-sylvo-pastoraux et la société rurale en Margeride. Doc. Station de Recherches sur la Forêt et l'Environnement, C.R.F., Ardon, 45160 Olivet.

NyS C., 1973. Les sols du plateau de Millevaches. Sci. Sol, A.F.E.S., n ${ }^{\circ} 4$.

Oswald H., 1969. Conditions forestières et potentialité de l'Epicéa en Haute Ardèche. Ann. Sci. for., 26 (2), 183-224.

STAGE A.R., 1976. An expression for the effect of aspect, slope, and habitat type on tree growth. For. Sci., 22 (4), 457-460. 